\title{
Patterns of Molecular Diversity in Current and Previously Developed Hybrid Parents of Pearl Millet [Pennisetum glaucum (L.) R. Br.]
}

\author{
Shashi Kumar Gupta', Thirunavukkarasu Nepolean², S. Mukesh Sankar², \\ Abhishek Rathore ${ }^{1}$, Roma Rani Das ${ }^{1}$, Kedar Nath Rai ${ }^{1}$, Charles Thomas Hash ${ }^{3}$ \\ ${ }^{1}$ International Crops Research Institute for the Semi-Arid Tropics (ICRISAT), Patancheru, India \\ ${ }^{2}$ Indian Agricultural Research Institute (IARI), New Delhi, India \\ ${ }^{3}$ International Crops Research Institute for the Semi-Arid Tropics (ICRISAT), Niamey, Niger \\ Email: s.gupta@cgiar.org
}

Received 17 April 2015; accepted 12 July 2015; published 15 July 2015

Copyright (C) 2015 by authors and Scientific Research Publishing Inc.

This work is licensed under the Creative Commons Attribution International License (CC BY). http://creativecommons.org/licenses/by/4.0/

\section{Open Access}

\section{Abstract}

ICRISAT's pearl millet (Pennisetum glaucum (L.) R. Br.) breeding program at Patancheru, India, has developed genetically diverse hybrid parents since 1980s. The present study investigated genetic diversity pattern between two groups of parents in this program, bred till 2004 and developed during 2004-2010. Combined analysis of 379 hybrid parents (current 166 parents and 213 previously developed hybrid parents) carried out using a set of highly polymorphic 28 SSRs detected 12.7 alleles per locus. An average of 8.5 and 8.7 SSR alleles per locus were found in previously developed and current parents, respectively, indicating marginal improvement in the levels of genetic diversity of hybrid parents in this program. Distance matrix differentiated these current and previously developed hybrid parents into 2 separate clusters, indicating infusion of new genetic variability over time as reflected by development of more genotype-specific alleles. Also, the seed and restorer parents were found clearly separated from each other in both the sets with few crossovers, indicating existence of two diverse and broad-based pools in hybrid parents of pearl millet. Restorer parents (R-lines) were found more diverse than seed parents (B-lines), as higher average gene diversity was detected among $R$-lines $(0.70)$ than $B$-lines $(0.56)$, though variation between $B$ and $\mathrm{R}$-lines was found reduced in newly developed lines to $9.22 \%$ from $16.98 \%$ in previously developed lines. Results suggested that newly developed lines were as much divergent when compared with previously developed lines, indicating that current ICRISAT pearl millet breeding program was moving towards development of diverse new hybrid parental lines. The study suggested use of trait-specific donors in B- and R-lines separately to maintain sufficient genetic distance between seed and restorer breeding lines. It was pointed out to cross parents having higher genetic distance within the seed (B-lines) and restorer (R-lines) breeding programs to derive diverse and productive hybrid parental lines in future. 


\section{Keywords}

\section{Pearl Millet, Simple Sequence Repeat, Hybrid Parents, Heterotic Pool, Genetic Diversity}

\section{Introduction}

Pearl millet (Pennisetum glaucum (L.) R. Br.) is cultivated on about 30 million ha in more than 30 countries with the majority of this area in Asia ( $>10$ million ha), Africa (about 18 million ha), and $>2$ million ha in the Americas. It is important as a high temperature and drought tolerant grain crop in Africa and Asia, particularly on low-fertility soils with limited water and nutrient holding capacity and is equally important as a hot season forage crop in semi-arid and arid parts of the world [1]. In Americas, it is used primarily as temporary summer pasture or as mulch in no-till soybean production systems [2]. India is the largest producer of this crop with 9.6 million ha area and 10 million tons of grain production [3]. The productivity of pearl millet in India rose from $305 \mathrm{~kg} \cdot \mathrm{ha}^{-1}$ during pre-hybrid era (1951-1955) to $1040 \mathrm{~kg} \cdot \mathrm{ha}^{-1}$ during present hybrid dominated era (2008-2013), and registered $227 \%$ improvement [4]. Hybrid technology contributed significantly to this increase in grain yield performance in India, and currently about 70\% of the pearl millet area in India is sown to hybrids. Based on the expected significant contribution of hybrids to pearl millet productivity enhancement in India, in the 1980s ICRISAT-Patancheru re-aligned its research strategy to better complement those of the Indian national program and private seed industry, and so focused on genetic diversification of hybrid parents. A recently conducted study found that private seed companies developed 103 hybrids in India during 2000-2010, and 62 (60\%) were directly or indirectly based on ICRISAT bred hybrid parents [5].

Genetic diversification of hybrid parents has been the key to developing the diverse range of hybrids required to adapt under different agro-ecologies in India. Hence, ICRISAT-Patancheru's pearl millet hybrid breeding program utilizes new genetic stocks regularly in the crossing program to widen the genetic diversity of hybrid parents. Hence, in the late 1990s and early 2000 years, there was an increase in the use of already designated hybrid parents and newly developed composites in ICRISAT pearl millet hybrid parent's development crossing program to derive new wave of genetically diverse inbreds. In this breeding process, it is important to monitor the levels of genetic diversity among these newly developed promising hybrid parents, and SSRs being environment independent multi-allelic genomic tools offer considerable advantages for this. Hence, the present study was undertaken to assess the genetic diversity in 166 newly developed hybrid parents (yet to be designated 88 B-lines and 78 R-lines, drawn from ICRISAT-Patancheru's hybrid breeding program in 2010) using a subset of highly polymorphic 28 SSR loci as suggested by previous studies [6]-[11] and results were compared with those for 213 previously developed hybrid parents (98 B-lines and 113 R-lines, developed during 1981 to 2004) to provide information on trends for genetic diversity in this breeding program over time.

\section{Materials and Methods}

\subsection{Plant Material}

Two sets of hybrid parents, the first set (set-I of 213 hybrid parental lines comprising of 98 seed parents and 115 restorer parents, designated between 1984 to 2004 at ICRISAT-Patancheru), and a second set (set-II) of 166 current hybrid parents comprising of 88 seed parents (numbered from B-1 to B-88) and 78 restorer parents (numbered from R-89 to R-166) bred by the hybrid parent breeding program of ICRISAT-Patancheru and available in 2010, were used in the present study. The set-I parents were characterized for morphological traits [12] [13] and using molecular markers [14]. Tift $23 \mathrm{D}_{2} \mathrm{~B}_{1}$, used as a reference genotype, was bred at the Coastal Plain Experiment Station, Tifton, GA, by introducing the $d_{2}$ dwarfing gene into the genetic background of an elite line Tift $23 \mathrm{~B}_{1}$, which is a maintainer of the $\mathrm{A}_{1}$ cytoplasmic-nuclear male sterility (CMS) system [15].

\subsection{DNA Extraction}

The B- and R-lines along with Tift $23 \mathrm{D}_{2} \mathrm{~B}_{1}$ were grown in small plastic pots in a greenhouse. Approximately 30 mg of leaf tissue from 20 - 25 seedlings (14 d old) were collected and bulked after removing the leaf tips and midribs. The harvested leaf samples were immediately collected in 96-well plate that consisted of 95 lines and 
one control (Tift 23D $\mathrm{B}_{1}$ ). Genomic DNA was extracted following the standard protocol [16]. Quantification of DNA, quality check and normalization up to $5 \mathrm{ng} \cdot \mathrm{Hl}^{-1}$ were done on agarose gel (1.2\%, containing ethidium bromide).

\subsection{Molecular Markers}

A set of 23 genomic- and five expressed sequence tag-simple sequence repeat (SSR) primer pairs were selected (based on the high level of PIC detected in earlier studies), to genotype the B- and R-lines of both the sets (set-I and set-II) using an ABI 3700 DNA fragment analyzer (Applied Biosystems). Twenty-five of these 28 SSRs mapped on pearl millet genome: 4 to 7 SSR loci on linkage groups (LGs) 1, 2, 3, and 7; and 2 SSR loci each on LGs 5 and 6 [6] [17].

\subsection{Polymerase Chain Reaction (PCR) and Genotyping}

Polymerase chain reactions (PCRs) were performed in $5 \mu \mathrm{l}$ volumes in 384-well PCR plates (AB Gene Rochester). Each PCR reaction mixture contained $5 \mathrm{ng}$ of genomic DNA, $2 \mathrm{pmol} / \mu \mathrm{l}$ of each primer, $25 \mathrm{mM} \mathrm{MgCl} 2,2$ $\mathrm{mM}$ of each deoxyribonucleotide triphosphate, $10 \times$ reaction buffer, and $0.2 \mathrm{U}$ Amplitaq Gold Polymerase (Applied Bio-systems, India). After one denaturing step of $15 \mathrm{~min}$ at $94^{\circ} \mathrm{C}$, a touchdown amplification program was performed on GeneAmp 9700 thermal cycler (Applied Biosystems). This profile consisted of a denaturing step of $25 \mathrm{sec}$ at $94^{\circ} \mathrm{C}$ and an extension step of $30 \mathrm{sec}$ at $72^{\circ} \mathrm{C}$. The initial annealing step was 20 sec at $64^{\circ} \mathrm{C}$ for one cycle and subsequently the temperature was reduced by $1^{\circ} \mathrm{C}$ for every cycle until a final temperature of $55^{\circ} \mathrm{C}$ was reached. The annealing temperature of $55^{\circ} \mathrm{C}$ was maintained for the last 35 cycles of the amplification, followed by the final extension of $72^{\circ} \mathrm{C}$ for $7 \mathrm{~min}$. PCR products were pooled post-PCR, which contained $1 \mu \mathrm{l}$ of each of dye-labeled (FAM, VIC, NED and PET) product, $7 \mu \mathrm{l}$ of formamide, $0.3 \mu \mathrm{l}$ of the LIZ-labeled [500 $(-250)]$ size standard, and $4.2 \mu \mathrm{l}$ of distilled water. The DNA fragments were size-separated on ABI 3700 automatic DNA sequencer (Perkin-Elmer/Applied Biosystems). GeneScan 3.1 [18] was used to size peak patterns by using the internal LIZ-labeled (500 [-250]) size standard and Genotyper 3.1 [19] was used for allele calling. Final bins for individual marker were assigned with the help of marker repeat length using AlleloBin 2.0 program [20] and the binned data was used for further data analysis.

\subsection{Data Analysis}

Genotypic data for the 28 common SSR markers was analyzed collectively for all the 379 lines (213 lines of set-I and 166 lines of set-II), and also separately for set-I and set-II lines. PIC; allelic richness as determined by total number of alleles, alleles per locus, and occurrence of common, most frequent, rare, and unique alleles; gene diversity; and heterozygosity were estimated using the PowerMarker V3.0 software [21]. Unique alleles are those that are present in one line but absent in other lines. Rare alleles are defined as those whose frequency is $\leq 1 \%$ in the investigated materials. Common alleles are defined as those whose frequency is $1 \%$ to $20 \%$ while the most frequent alleles are those whose frequency is $>20 \%$. This classification of alleles was done following method used by other workers [22] [23].

AMOVA was performed [24] for both the sets individually and also pooled analysis to estimate the variance components among and within B- and R-line groups. The F value-the fixation index (or Wright's F statistic) (Fst) - from the AMOVA analysis provided a measure of genetic differentiation of the sub-groups. A simple matching allele frequency-based distance matrix was used in the DARwin-5.0 program [25] to construct a tree diagram to examine the genetic structure and diversity among B- and R-lines. The grouping of B- and R-lines into clusters and sub-clusters was done at $5 \%$ dissimilarity level.

\section{Results}

In the combined set of 379 hybrid parents, comprising of both previously designated (hereafter, referred to as set-I) as well as current (hereafter, referred as set-II) pearl millet hybrid parents, 355 alleles were detected by 28 SSR loci, with an average of 12.68 alleles per locus. The number of alleles per locus varied from 4 (Xpsmp2202, Xpsmp2246 \& Xipes3048) to 40 (Xpsmp2218), with ten to twenty alleles at 15 of the 28 SSR loci (Table 1). Marker Xpsmp2218 had up to 40 alleles while Xpsmp2068, Xpsmp2079 and Xpsmp2218 amplified 23 to 40 alleles per locus. The allele size range across the loci and lines varied from 4 bp (Xpsmp2201) to 121 bp 
(Xpsmp2079). Polymorphic information content, (PIC) ranged from 0.26 (Xpsmp2222) to 0.93 (Xpsmp2218) with an average of 0.67 . Of the 28 SSRs used, 25 were moderately polymorphic (PIC $>0.44$ ), of which 12 were highly polymorphic (PIC $>0.70$ ) with PIC values ranging from 0.70 to 0.93 . Furthermore, the average PIC values for B- and R-lines were 0.56 and 0.70 , respectively. Gene diversity, defined as the probability that two randomly chosen alleles from the population are different, varied from 0.28 (Xpsmp2222) to 0.93 (Xpsmp2218) with an average of 0.70 . Restorer lines (R-lines) had higher average gene diversity (0.73) than B-lines (0.60). The level of heterozygosity in SSRs across B- and R-lines ranged from 0.01 to 0.07 (excluding for Xpsmp2077 which had high value of 0.12 ) and averaging 0.04 , with $>0.05$ in eight SSRs and $>0.10$ in one SSR. The R-lines had higher average heterozygosity (0.05) than B-lines (0.03). Moreover, ICMB 96666, B-23, B-24, B-57, B-65, B-6, B-45 and B-46 among B-lines; and ICMP 451, IPC 337, IPC 492, IPC 962, IPC 997, IPC 1018, IPC 1027, IPC 1078, IPC 1307, IPC 1503, IPC 1617, R-118, R-125, R-128, R-131, R-144, R-148, R-155, R-159, R-160, and R-166 among R-lines were genetically more variable than other lines, with heterozygosity at four to eight SSR loci detected each in the B-lines and in the R-lines (data not presented). Allele size for the internal control

Table 1. Allelic composition, polymorphic information content (PIC), gene diversity, and observed heterozygosity of the 28 simple sequence repeat (SSR) loci in combined set of pearl millet (186 B-lines and 193 R-lines).

\begin{tabular}{|c|c|c|c|c|c|c|c|c|}
\hline SSR loci & $\begin{array}{c}\text { Allele } \\
\text { richness }\end{array}$ & $\begin{array}{l}\text { Allele size } \\
\text { range (bp) }\end{array}$ & $\begin{array}{l}\text { Rare } \\
\text { alleles } \\
(\leq 1 \%)\end{array}$ & $\begin{array}{l}\text { Common } \\
\text { alleles } \\
\text { (1 to } \leq 20 \%)\end{array}$ & $\begin{array}{c}\text { Most } \\
\text { frequent } \\
\text { alleles (>20\%) }\end{array}$ & $\mathrm{PIC}^{\dagger}$ & Gene diversity $^{\dagger}$ & Heterozygosity $^{\dagger}$ \\
\hline Xpsmp2045 & 10 & $21(195-216)^{\ddagger}$ & 7 & 1 & 2 & $0.51(0.37,0.54)$ & $0.59(0.41,0.60)$ & $0.02(0.01,0.03)$ \\
\hline Xpsmp2068 & 23 & $42(99-141)$ & 10 & 12 & 1 & $0.87(0.76,0.89)$ & $0.88(0.78,0.90)$ & $0.03(0.04,0.02)$ \\
\hline Xpsmp2077 & 9 & $40(138$ - 178) & 3 & 4 & 2 & $0.51(0.47,0.43)$ & $0.58(0.56,0.46)$ & $0.12(0.08,0.15)$ \\
\hline Xpsmp2079 & 33 & $121(138-259)$ & 11 & 22 & 0 & $0.91(0.85,0.94)$ & $0.92(0.86,0.94)$ & $0.04(0.02,0.06)$ \\
\hline Xpsmp2089 & 20 & $30(103-133)$ & 4 & 15 & 1 & $0.88(0.78,0.91)$ & $0.89(0.81,0.92)$ & $0.05(0.07,0.04)$ \\
\hline Xpsmp2090 & 10 & $20(171-191)$ & 2 & 6 & 2 & $0.74(0.70,0.77)$ & $0.78(0.74,0.79)$ & $0.008(0.01,0.01)$ \\
\hline Xpsmp2201 & 10 & $4(332-336)$ & 2 & 6 & 2 & $0.66(0.50,0.75)$ & $0.70(0.59,0.78)$ & $0.02(0.01,0.03)$ \\
\hline Xpsmp2202 & 4 & $18(145$ - 163) & 0 & 1 & 3 & $0.59(0.59,0.59)$ & $0.66(0.67,0.65)$ & $0.03(0.02,0.04)$ \\
\hline Xpsmp2203 & 19 & 37 (335 - 372) & 4 & 13 & 2 & $0.86(0.66,0.91)$ & $0.87(0.70,0.92)$ & $0.03(0.01,0.06)$ \\
\hline Xpsmp2204 & 11 & $94(173-267)$ & 3 & 7 & 1 & $0.58(0.26,0.77)$ & $0.60(0.30,0.80)$ & $0.02(0.01,0.03)$ \\
\hline Xpsmp2209 & 12 & $23(337-360)$ & 4 & 5 & 3 & $0.82(0.79,0.79)$ & $0.84(0.81,0.81)$ & $0.07(0.04,0.10)$ \\
\hline Xpsmp2218 & 40 & $59(217$ - 276) & 19 & 21 & 0 & $0.93(0.87,0.94)$ & $0.93(0.88,0.94)$ & $0.03(0.01,0.05)$ \\
\hline Xpsmp2220 & 12 & $24(108$ - 132) & 4 & 7 & 1 & $0.70(0.41,0.83)$ & $0.72(0.43,0.85)$ & $0.04(0.01,0.07)$ \\
\hline Xpsmp2222 & 5 & $8(152$ - 160) & 0 & 4 & 1 & $0.26(0.23,0.30)$ & $0.28(0.24,0.31)$ & $0.05(0.08,0.02)$ \\
\hline Xpsmp2227 & 5 & 15 (194 - 209) & 2 & 2 & 1 & $0.28(0.25,0.30)$ & $0.31(0.28,0.34)$ & $0.02(0.01,0.03)$ \\
\hline Xpsmp2232 & 16 & $24(226-250)$ & 7 & 7 & 2 & $0.77(0.65,0.78)$ & $0.80(0.69,0.80)$ & $0.02(0.02,0.02)$ \\
\hline Xpsmp2237 & 10 & $56(202-258)$ & 5 & 4 & 1 & $0.56(0.21,0.73)$ & $0.60(0.22,0.77)$ & $0.04(0.03,0.04)$ \\
\hline Xpsmp2246 & 4 & $6(258-264)$ & 0 & 2 & 2 & $0.68(0.56,0.70)$ & $0.73(0.63,0.75)$ & $0.03(0.03,0.02)$ \\
\hline Xpsmp2248 & 6 & $10(162-172)$ & 0 & 4 & 2 & $0.70(0.73,0.64)$ & $0.74(0.77,0.69)$ & $0.02(0.03,0.02)$ \\
\hline Xpsmp2249 & 6 & $31(129-160)$ & 2 & 3 & 1 & $0.39(0.26,0.49)$ & $0.43(0.28,0.55)$ & $0.02(0.01,0.03)$ \\
\hline Xpsmp2273 & 19 & $62(158-220)$ & 9 & 9 & 1 & $0.77(0.71,0.79)$ & $0.78(0.73,0.81)$ & $0.04(0.02,0.05)$ \\
\hline Xipes3002 & 10 & $14(197$ - 211) & 4 & 5 & 1 & $0.65(0.63,0.65)$ & $0.68(0.67,0.69)$ & $0.05(0.02,0.07)$ \\
\hline Xipes3032 & 6 & $15(180$ - 195) & 2 & 2 & 2 & $0.63(0.57,0.57)$ & $0.68(0.63,0.64)$ & $0.07(0.05,0.08)$ \\
\hline Xipes3048 & 4 & $6(244-250)$ & 0 & 2 & 2 & $0.49(0.47,0.50)$ & $0.57(0.56,0.57)$ & $0.008(0.017,0)$ \\
\hline Xipes3080 & 7 & $15(211$ - 226) & 3 & 2 & 2 & $0.63(0.57,0.58)$ & $0.68(0.62,0.64)$ & $0.05(0.03,0.04)$ \\
\hline Xipes3088 & 11 & $24(144-168)$ & 2 & 8 & 1 & $0.83(0.77,0.80)$ & $0.85(0.80,0.82)$ & $0.03(0.05,0.01)$ \\
\hline CTM10 & 17 & $32(167$ - 199) & 3 & 13 & 1 & $0.78(0.46,0.91)$ & $0.80(0.50,0.91)$ & $0.05(0.01,0.10)$ \\
\hline CTM12 & 16 & $25(318$ - 343) & 5 & 9 & 2 & $0.75(0.64,0.77)$ & $0.78(0.67,0.80)$ & $0.03(0.04,0.02)$ \\
\hline Total & 355 & & 117 & 196 & 42 & & & \\
\hline Mean & 12.68 & & 4.18 & 7 & 1.5 & $0.67(0.56,0.70)$ & $0.70(0.60,0.73)$ & $0.04(0.03,0.05)$ \\
\hline
\end{tabular}

${ }^{\dagger} \mathrm{PIC}$, gene diversity, and heterozygosity for the B- and R-lines are given in parentheses, respectively; ${ }^{\ddagger}$ Numbers in parentheses indicate range. 
(Tift 23D $\mathrm{D}_{2}$ ) were uniform and reproducible for each of the markers indicating the accuracy of the protocol and reproducibility of allelic data for a given primer across assays.

Of the 355 alleles detected in B- and R-lines, 117 were rare (frequency $\leq 1 \%$ ), 196 common (frequency $1 \%$ to 20\%), and 42 most frequent (frequency $>20 \%$ ) alleles (Table 1), with more alleles observed in R-lines (329 alleles in 193 lines averaging 1.70 alleles per line) than the B-lines (237 alleles in 186 lines averaging 1.27 alleles per line). "Most frequent" alleles were detected at all the SSR loci (except in SSR loci, Xpsmp2079 and Xpsmp2218), ranging from one to three and averaging 1.5 alleles per locus. The rare alleles ranged from 0 (Xpsmp2202, Xpsmp2222, Xpsmp2246, Xpsmp2248 and Xipes3048) to 19 (Xpsmp2218), averaging 4.18, while the common alleles ranged from 1 (Xpsmp2045 and Xpsmp2202) to 22 (Xpsmp2079), averaging 7.0. Twenty-one SSR loci detected all three (rare, common, and most frequent) classes of alleles (Table 1). Genotype-specific alleles were detected in $15 \mathrm{~B}$-and $37 \mathrm{R}$-lines (Table 2).

The combined Analysis of Molecular Variance (AMOVA) for all the lines showed significant differences between set-I and set-II, which accounted for $19.20 \%$ of the total genetic variation (Table 3). Also, significant

Table 2. Genotype-specific alleles present in 15 seed parents (B-lines) and 37 restorer parents (R-lines) of pearl millet (from both the sets).

\begin{tabular}{|c|c|c|c|}
\hline Accession identity $^{\dagger}$ & SSR allele ${ }^{\ddagger}$ & Accession identity & SSR allele \\
\hline \multicolumn{4}{|c|}{ Seed parents (B-lines) } \\
\hline ICMB 91444 & Xpsmp2209 ${ }_{353}$ & B-13 & Xpsmp2079 ${ }_{138}$ \\
\hline ICMB 91666 & Xpsmp2079 259 & B-33 & Xpsmp2218 234 \\
\hline ICMB 92444 & Xpsmp2218 217 & B-37 & Xpsmp2045 216 \\
\hline ICMB 96222 & Xpsmp2068 ${ }_{137}$ & B-44 & Xpsmp2218 276 \\
\hline \multirow[t]{6}{*}{ ICMB 98333} & Xpsmp2232 226 & B-59 & Xipes3002 208 \\
\hline & & B-49 & Xpsmp2273 188 \\
\hline & & B-61 & Xpsmp2227 197 \\
\hline & & B-63 & Xpsmp2237 234 \\
\hline & & B-64 & Xpsmp2068 102 \\
\hline & & B-66 & Xpsmp2218 268 \\
\hline \multicolumn{4}{|c|}{ Restorer parents (R-lines) } \\
\hline IPC 244 & Xpsmp2204 ${ }_{191}$ & $\mathrm{R}-94$ & Xpsmp2273 186 \\
\hline IPC 338 & CTM10 169 & R-96 & Xpsmp $2237_{202}$ and $X p s m p 2273_{194}$ \\
\hline IPC 367 & Xpsmp2203 343 & R-98 & Xpsmp2045 203 \\
\hline IPC 487 & Xpsmp2204 ${ }_{175}$ & $\mathrm{R}-100$ & Xpsmp2079 224 \\
\hline IPC 632 & Xpsmp2220 132 & $\mathrm{R}-103$ & Xpsmp2201 2445 \\
\hline IPC 687 & Xpsmp2232 242 & $\mathrm{R}-121$ & Хрsmp2218 238 \\
\hline IPC 689 & CTM12 $2_{335}$ & $\mathrm{R}-122$ & Xpsmp2089 111 \\
\hline IPC 774 & Xpsmp2232 250 & $\mathrm{R}-131$ & Xpsmp2218 270 \\
\hline IPC 821 & Xpsmp2203 361 & $\mathrm{R}-136$ & Xpsmp2045 209 \\
\hline IPC 962 & Xpsmp2273 ${ }_{174}$ & $\mathrm{R}-138$ & Xpsmp2218 250 \\
\hline IPC 1027 & Xpsmp2218 247 & $\mathrm{R}-140$ & Xpsmp2209 ${ }_{342}$ \\
\hline IPC 1047 & Xpsmp2077 142 & $\mathrm{R}-141$ & Xpsmp2079 215 \\
\hline IPC 1114 & Xpsmp2220 ${ }_{114}$ & $\mathrm{R}-146$ & Xpsmp2089 ${ }_{132}$ \\
\hline IPC 1178 & Xpsmp2249 150 & $\mathrm{R}-149$ & Xipes3088 ${ }_{168}$ \\
\hline IPC 1254 & Xpsmp2273 176 & $\mathrm{R}-158$ & CTM12 $2_{324}$ \\
\hline IPC 1307 & Xpsmp2079 235 & & \\
\hline IPC 1329 & Xpsmp2273 ${ }_{180}$ & & \\
\hline IPC 1351 & Xpsmp2068 129 & & \\
\hline IPC 1445 & Xpsmp2079 219 & & \\
\hline IPC 1536 & CTM10 199 & & \\
\hline
\end{tabular}

${ }^{\dagger}$ ICMB, ICRISAT millet B-line; IPC, ICRISAT pollinator (restorer) collection; ${ }^{\ddagger}$ SSR, simple sequence repeat. 
differences were observed between B- and R-line groups within each set, which accounted for $11.55 \%$ of the total genetic variation. The within-group variation, both in the B-lines and R-lines, was much larger and accounted for $69.24 \%$ of the total observed genetic variation. Set-wise AMOVA showed higher variation between B- and R-line groups in set-I (16.98\%) than in set-II (9.92\%), as detected by 28 SSR loci. The fixation index (Fst) between B- and R-lines for the individual markers varied from 0.138 (Xpsmp2222) to 0.456 (Xpsmp2218) and was significant for all 28 SSR primer pairs (information not presented). Some of the markers such as Xipes3088, Xpsmp2209, Xpsmp2203, Xpsmp2068, Xpsmp2089, Xpsmp2079 and Xpsmp2218 had Fst values greater than 0.40 , contributed most to the variation between B- and R-line groups.

Mean for number of alleles per locus, gene diversity, heterozygosity and PIC for set-I using the set of 28 SSRs was 8.50, 0.63 (0.50 for B-lines and 0.63 for R-lines), 0.03 (0.02 for B-lines and 0.04 for R-lines), and 0.59 ( 0.46 for B-lines and 0.60 for R-lines) respectively (Table 4). In set-II of current pearl millet hybrid parents, 28 SSR loci detected a total of 244 alleles among 88 B- and 78 R-lines, with an average of 8.7 alleles per locus, and PIC ranged from 0.17 (Xpsmp2203) to 0.91 (Xpsmp2089) with an average of 0.57; the average PIC values for B- and R-lines were 0.46 and 0.62, respectively; gene diversity varied from 0.19 (Xpsmp2204) to 0.91 (Xpsmp2089) with an average of 0.61 ; restorer lines (R-lines) had higher average gene diversity (0.66) than B-lines (0.50); and the R-lines had higher average heterozygosity (0.05) than B-lines (0.04). Of the 244 alleles detected in B- and R-lines, more alleles were observed in R-lines (222 alleles in 78 lines averaging 2.85 alleles per line) than the B-lines (163 alleles in 88 lines averaging 1.85 alleles per line).

The neighbor-joining (NJ) tree based on a simple matching dissimilarity matrix of both the sets clearly differentiated set-I hybrid parents from set-II hybrid parents (Figure 1) into two separate clusters. 11 B-lines (11/186) were found in the R-lines cluster, while 30 R-lines (30/193) were found in the B-lines cluster. The dissimilarity matrix for 166 set-II parents clearly differentiated the majority of B- and R-lines into two separate clusters (Figure 2).

Table 3. Analysis of molecular variance (AMOVA) of set-I, set-II and for combined sets using 28 SSRs.

\begin{tabular}{|c|c|c|c|c|c|}
\hline Source of variation & df & Sum of squares & Variance components & Percentage of variation & $\begin{array}{c}P(10,000 \\
\text { permutations })\end{array}$ \\
\hline \multicolumn{6}{|l|}{ Set I } \\
\hline Between B- and R-line groups & 1 & 256.4 & 1.18 & 16.98 & 0.00000 \\
\hline Within B- and R-line groups & 424 & 2454.8 & 5.78 & 83.02 & 0.00000 \\
\hline Total & 425 & 2711.2 & 6.97 & & \\
\hline \multicolumn{6}{|l|}{ Fixation index: 0.16981} \\
\hline \multicolumn{6}{|l|}{ Set II } \\
\hline Between B- and R-line groups & 1 & 93.2 & 0.53 & 9.22 & 0.00000 \\
\hline Within B- and R-line groups & 330 & 1726.6 & 5.23 & 90.78 & 0.00000 \\
\hline Total & 331 & 1819.8 & 5.76 & & \\
\hline \multicolumn{6}{|l|}{ Fixation index: 0.092} \\
\hline \multicolumn{6}{|l|}{ Combined for both sets } \\
\hline Between set-I and set-II & 1 & 777.8 & 1.58 & 19.20 & 0.00000 \\
\hline Between B- and R-line groups within sets & 2 & 371.8 & 0.95 & 11.55 & 0.00000 \\
\hline Among lines within groups & 754 & 4318.9 & 5.72 & 69.24 & 0.00467 \\
\hline Total & 757 & 5468.5 & 8.27 & & \\
\hline Fixation index: 0.30756 & & & & & \\
\hline
\end{tabular}

Table 4. Genetic diversity parameters of set-I and set-II lines using same set of the 28 simple sequence repeat (SSR) loci in pearl millet.

\begin{tabular}{|c|c|c|c|c|c|}
\hline SSR loci & Allele richness & Alleles per line ${ }^{\dagger}$ & $\mathrm{PIC}^{\dagger}$ & Gene diversity $^{\dagger}$ & Heterozygosity $^{\dagger}$ \\
\hline Set-I & 8.50 & $(1.84,2.02)$ & $0.59(0.46,0.60)$ & $0.63(0.50,0.63)$ & $0.03(0.02,0.04)$ \\
\hline Set-II & 8.70 & $(1.85,2.85)$ & $0.57(0.46,0.62)$ & $0.61(0.50,0.66)$ & $0.04(0.04,0.05)$ \\
\hline
\end{tabular}

${ }^{\dagger}$ Alleles per line, PIC, gene diversity, and heterozygosity for the B- and R-lines are given in parentheses, respectively. 


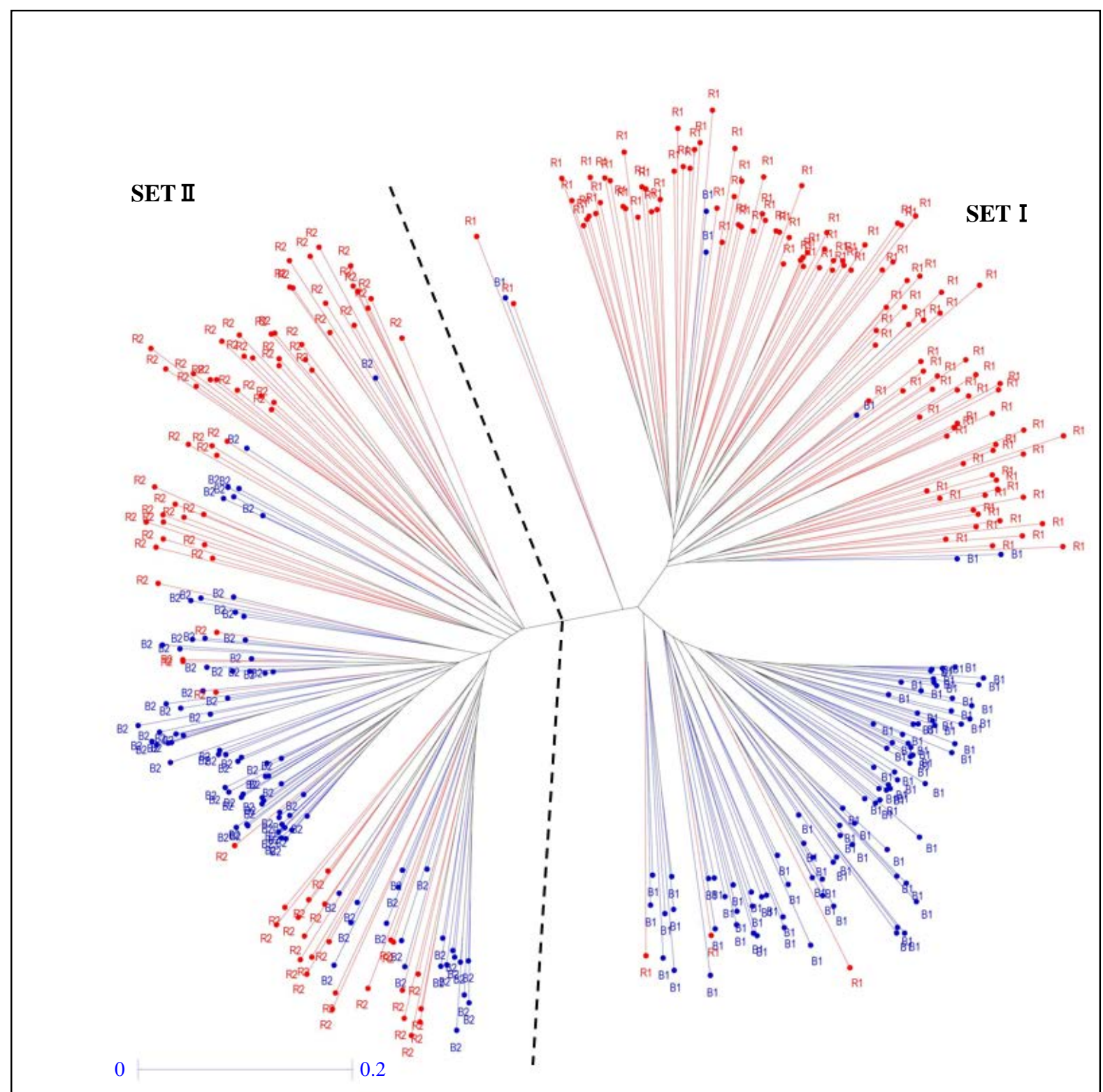

Figure 1. Unweighted neighbor-joining tree based on a simple matching dissimilarity matrix for allele sizes, detected by 28 simple sequence repeat primer pairs across 379 pearl millet hybrid parental lines (213 set-I lines and 166 set-II lines). Accessions are identified as "B" for seed parental lines and "R" as restorer lines. Suffix 1 and 2 with B- and R-lines represent set-I and set-II lines, respectively. B-lines are shown in blue and R-lines in red color.

Tree diagrams were also constructed separately for set-II B- and R-lines, which further had four sub-clusters each for B- and R-lines (Figure 3). However, 7 B-lines grouped within the R-line cluster and eighteen R-lines grouped with the B-line cluster for this set of lines. Nine of the $18 \mathrm{R}$-lines found in the B-line cluster had presence of seed parents (B-lines) in their parentage, of which 7 had the ICRISAT High Head Volume B-Composite (HHVBC) or its derived progeny (Table 5). Of the 7 B-lines found clustered with R-lines, 2 had ICTP 8202 in their parentage and one was derived from Nigerian Composite D2 Dwarf (NCD2) [26].

The 88 B-lines grouped into four sub-clusters, of which 22 lines were in cluster B-I, 15 in B-II, 24 in B-III and 27 in B-IV (Figure 3(a)). Fourteen lines having 843B in their parentage grouped in the B-I cluster. 843B is an early-maturing $d_{2}$-dwarf line developed from Kansas State University (KSU) breeding line BKM 2068 [27]. Furthermore, another early promising line, ICMB 89111 derived from a cross involving 843B, was present in the parentage of 3 lines of B-I cluster. This group also had 4 lines that had progenies of Extra Early B-Composite 


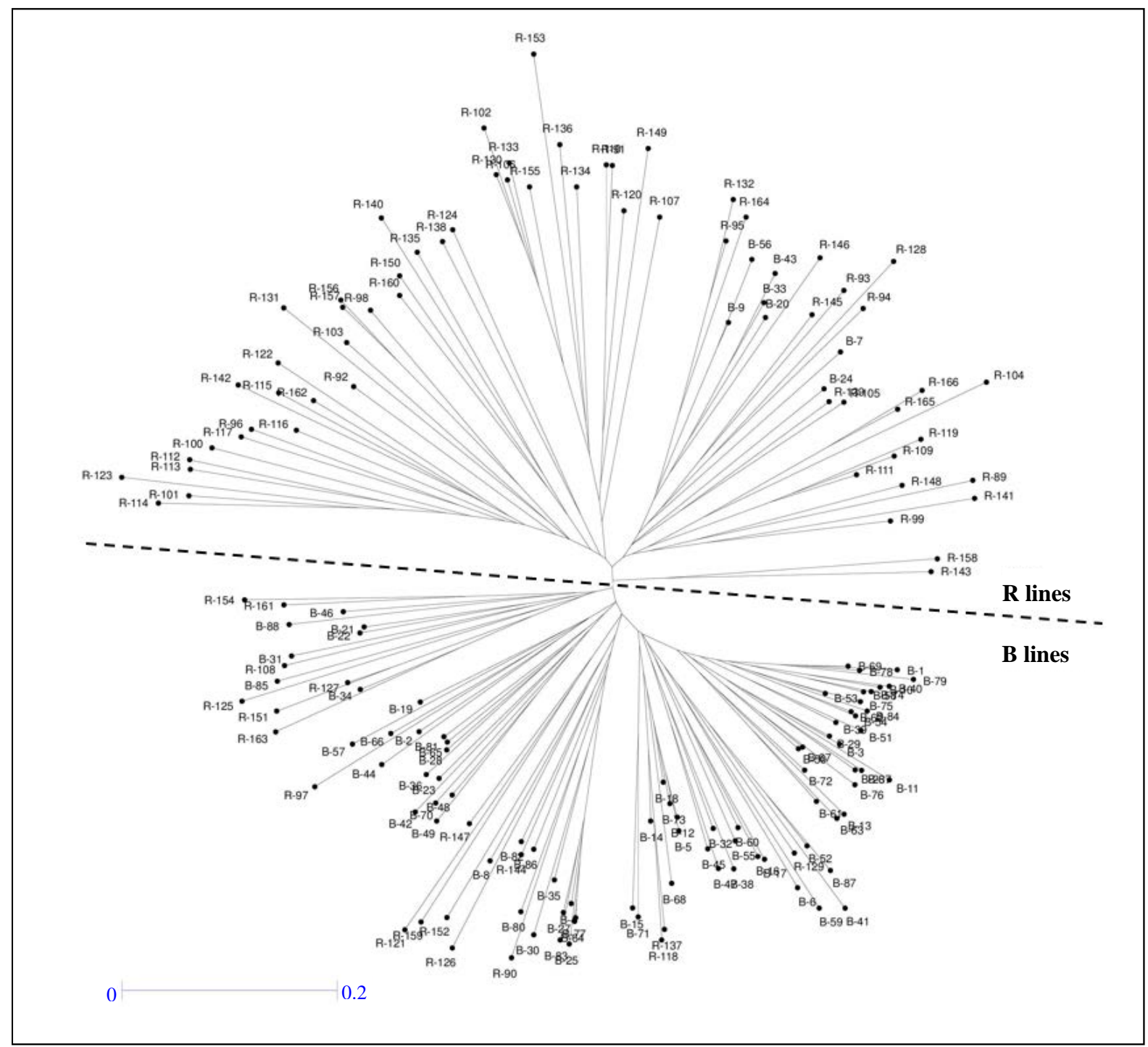

Figure 2. Unweighted neighbor-joining tree based on a simple matching dissimilarity matrix for allele sizes detected by 28 simple sequence repeat primer pairs across 88 seed parental lines (B-lines; B-1 to B-88) and 78 restorer lines (R-lines; R-89 to R-166) of pearl millet set-II.

(EEBC) in their parentage; EEBC is an extra-early-maturing and day-length insensitive maintainer composite developed using iniadi germplasm [28]. In cluster B-II, 7 lines had both 843B and 81B [29] in their parentage. Eleven lines, derived either directly from HHVBC or having its progeny in their parentage, were found very close in cluster B-III, along with 3 other such lines in this sub-cluster. Sub-cluster B-IV, with maximum number of 27 lines, had lines with mixed parentage. For instance, it had 8 lines with 843B, 13 lines with HHVBC progenies, and 5 lines with EEBC progenies in their parentages. Likewise, the 78 R-lines formed 4 sub-clusters (R-I, R-II, R-III and R-IV) comprised of 12, 8, 39, and 19 lines (Figure 3(b)). Four of the 8 lines in cluster R-II had lines derived from the ICRISAT Smut Resistant Composite (SRC) in their parentage. Of the 19 lines in cluster R-IV, 8 lines had progenies of MRC (Mandore Restorer Composite), and 5 lines had Rajasthan Composite Bajra (RCB) or Rajasthan Inbred Bajra (RIB) progenies in their parentage. Clusters R-I and R-III had lines with mixed pedigrees.

ICRISAT-bred B-lines designated between 1992 and 2004 were found in the parentage of 42 of the 88 B-lines in set-II. New pearl millet composites and improved breeding populations, like the ICRISAT Medium Composite 94 (MC 94), released composite variety AIMP 92901 (based on the ICRISAT Bold-Seeded Early Composite, 
BSEC), the ICRISAT Early Smut Resistant Composite II (ESRC II), released composite variety JBV 3 (based on the ICRISAT Smut Resistant Composite II, SRC II), released composite variety Raj 171 (based on the ICRISAT Intervarietal Composite, IVC), SDMV 90031, released variety GB 8735, SDMV 95045, released synthetic variety ICMS 7704, and several RCB varieties were found in parentages of 48 of the 77 set-II R-lines.

\section{Discussion}

\subsection{Genetic Diversity of Pearl Millet Hybrid Parents}

Enhancing the genetic diversity among hybrid parental lines is an essential component of hybrid breeding

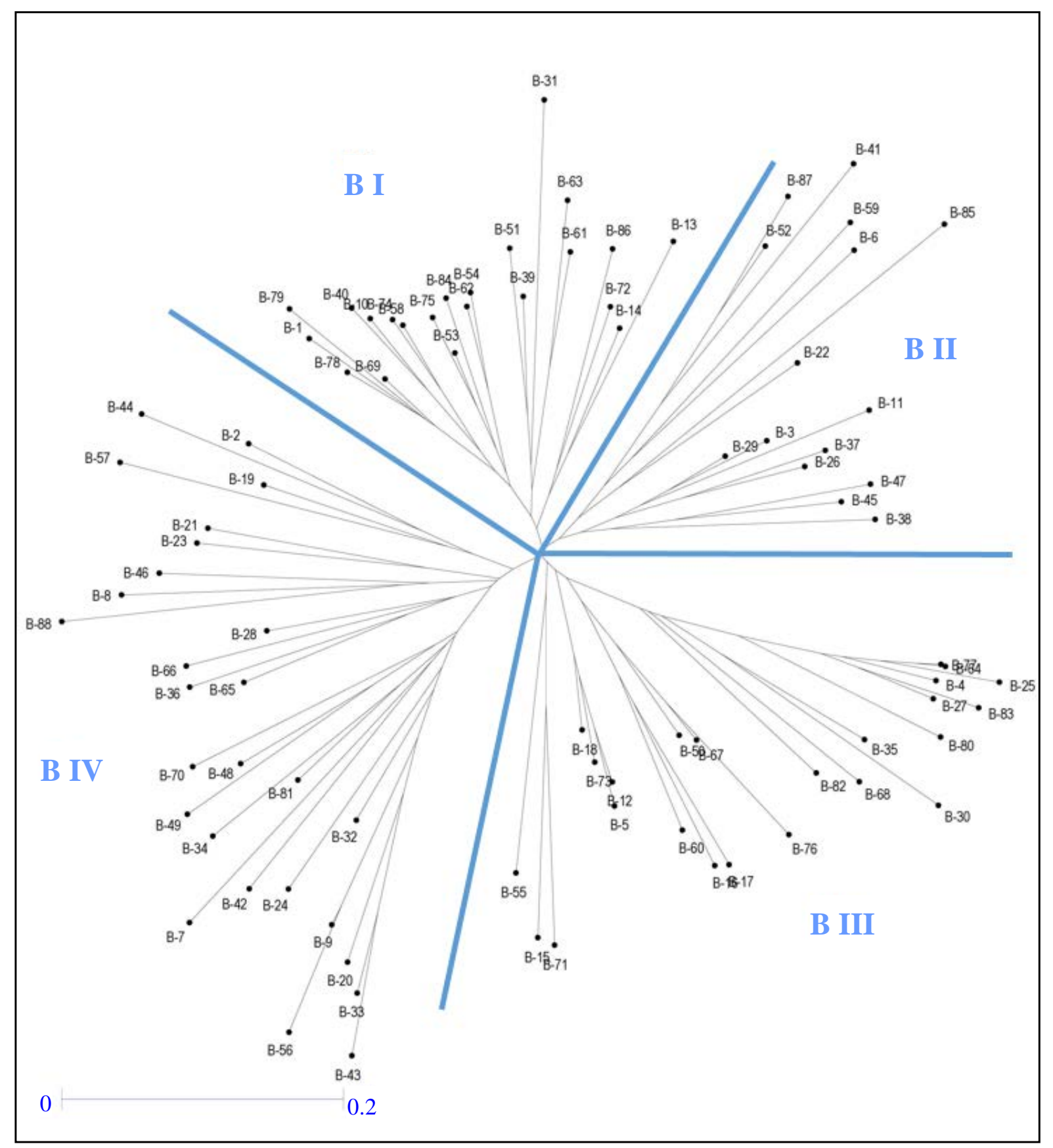




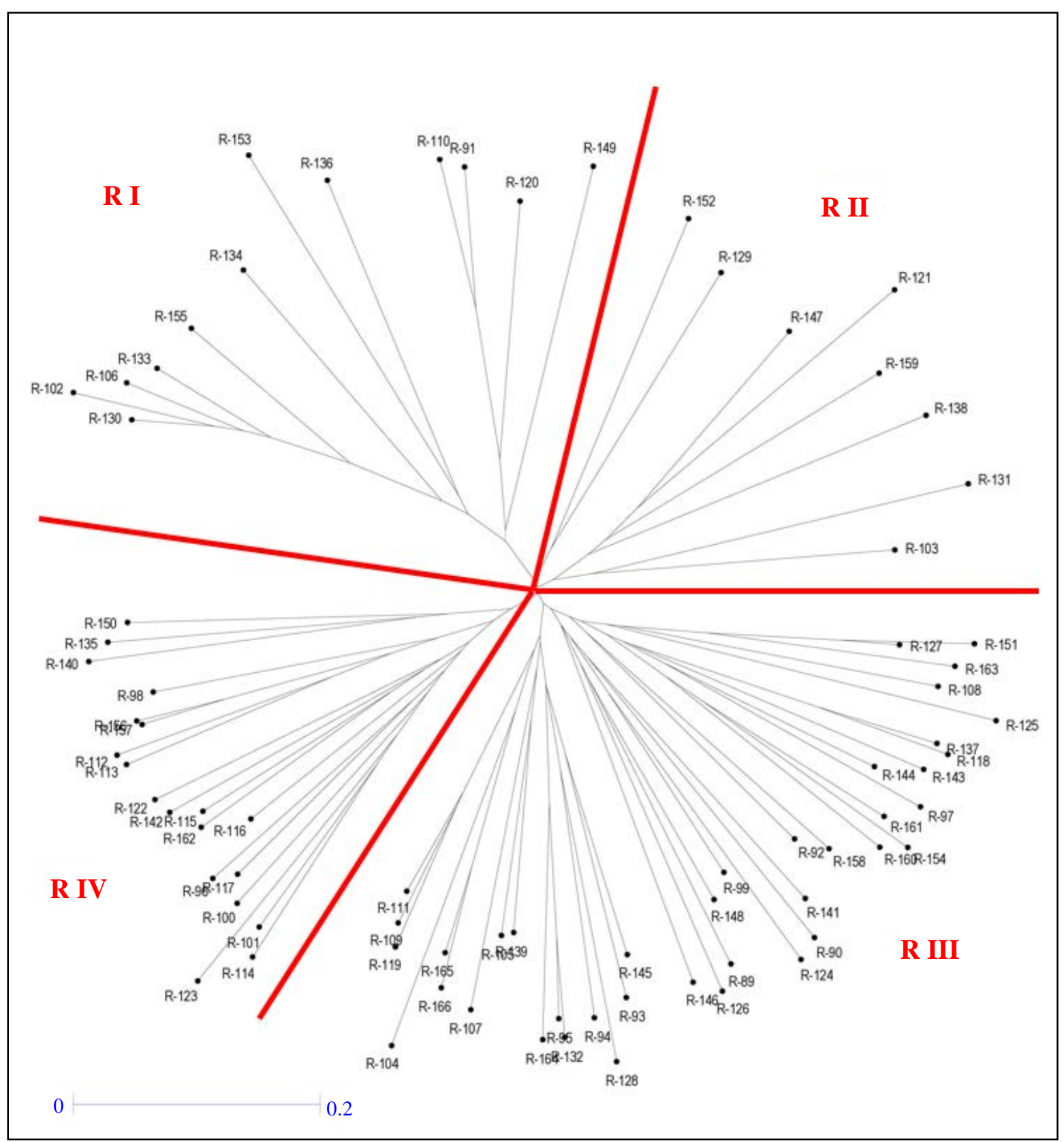

(b)

Figure 3. Un-weighted neighbor-joining tree based on a simple matching dissimilarity matrix for allele sizes detected by 28 simple sequence repeat primer pairs in set-II lines. (a) Across 88 B-lines (B-1 to B-88); and (b) Across 77 R-lines (R-89 to $\mathrm{R}-166)$.

programs to maximize heterosis for higher grain yields. Thus, the ICRISAT-Patancheru pearl millet hybrid parent breeding program utilizes different kinds of germplasm and breeding materials in its crossing program to diversify the genetic base of the hybrid parents it develops. In the present study, the combined clustering analysis of 379 hybrid parents (213 previously designated parents of set-I and 166 newly developed parents of set-II) based on Polymerase Chain Reaction (PCR) products detected by 28 SSR primer pairs, showed 12.68 alleles per locus. This value was higher than reported earlier on pearl millet hybrid parents using SSR markers. For instance, Kapila et al. [10] detected 6.26 alleles per primer pair among 72 inbred lines (primarily B-lines bred at ICRISAT-Patancheru); and Sumathi et al. [11] reported 2.76 alleles per primer pair among 42 inbred lines bred 
Table 5. Parentage of pearl millet set-II B- and R-lines found in alternate clusters (B-lines in R-lines cluster and vice versa) for set-II lines.

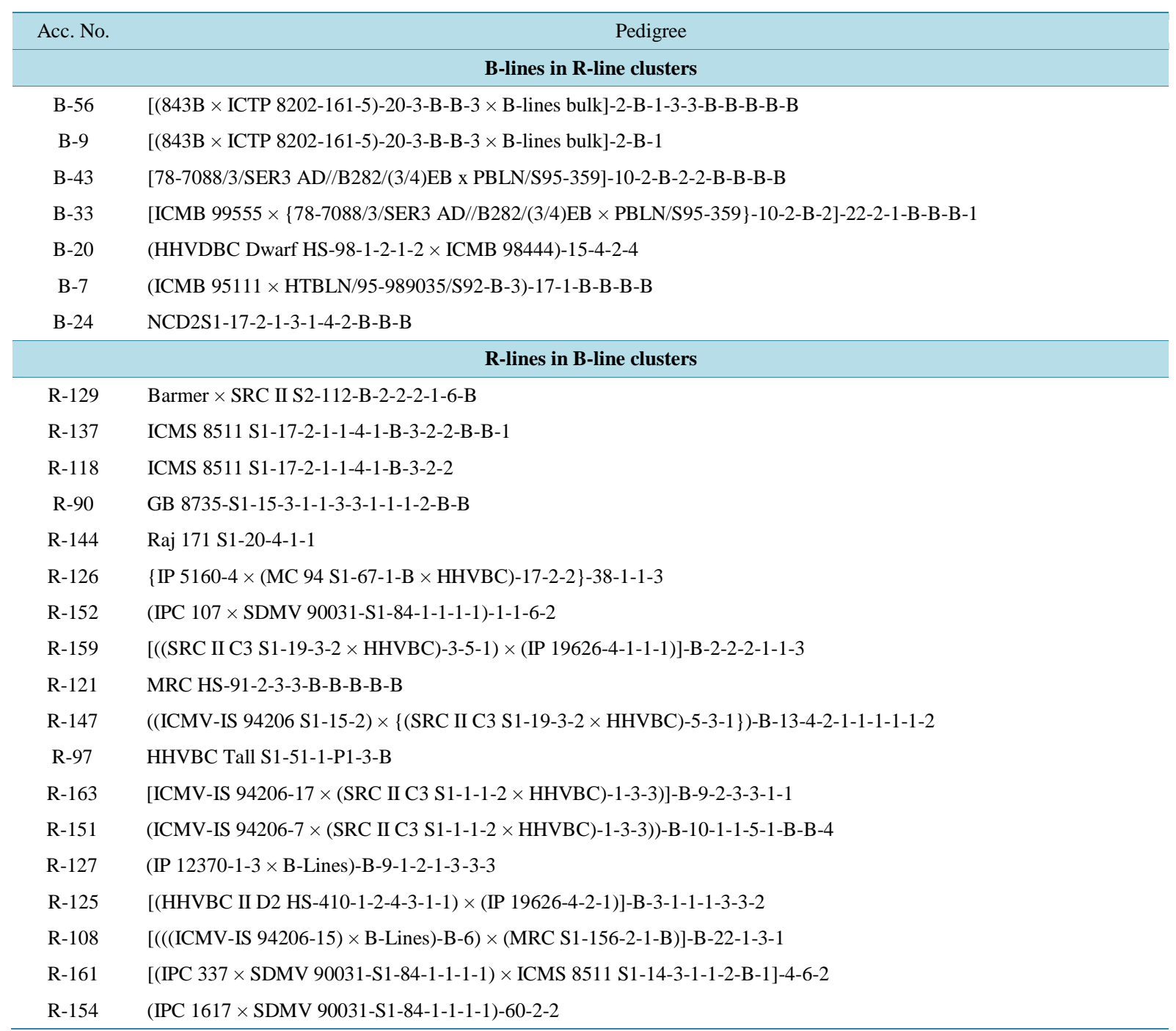

primarily at Tamil Nadu Agricultural University, Coimbatore, India. The involvement of higher number of lines (379) in the present study, in comparison to the two mentioned studies (with 72 and 42 lines), might be the reason of higher average number of allele per locus in this study. However, still higher numbers of alleles per SSR primer pair (16.4) were reported in a study using 20 SSRs [30] and involving 145 pearl millet inbreds derived from diverse landrace germplasm and improved open-pollinated varieties of West and Central African origin. Similar finding of higher alleles per locus was also reported in case of maize (Zea mays L.) [31], where average of 14.57 alleles per locus was found in a study on 1537 elite inbred lines with 359 SSR markers. Twenty-eight SSR primer pairs used in the present study detected higher average number of alleles among R-lines (1.70) than among B-lines (1.27). Also, both average PIC ( 0.60 for B-lines and 0.73 for R-lines) and averages gene diversity ( 0.56 for B-lines and 0.70 for R-lines) indicated R-lines to be more genetically diverse than B-lines, which might be due to the broader genetic base of germplasm used in the development of restorer lines.

\subsection{Genetic Diversity in Seed and Restorer Parents Bred over an Interval of Time}

Number of alleles per locus in set-II was 8.7 in comparison to 8.5 of set-I with the same set of 28 SSRs, indicating comparable genetic diversity in the hybrid parents of both the sets, and allowing us to conclude that genetic diversity within the current inbreds has not reduced compared to that in the previously designated hybrid parents. 
This established that the levels of genetic diversity are consistent in this breeding program over time. The dissimilarity matrix-based tree clearly differentiated set-I from set-II lines, indicating that new variability is being continuously infused into the ICRISAT-Patancheru's pearl millet breeding program. New genetic stocks are regularly used in the pearl millet breeding program at ICRISAT-Patancheru to increase the genetic diversity of hybrid parents in the development of B- and R-lines. In the late 1990s and early 2000 years, there was increase in the use of already designated hybrid parents and newly developed composites in crossing program to derive new wave of genetically diverse second cycle inbreds. This was reflected from the parentages of lines under study, as new composites and improved populations, like MC 94, AIMP 92901, ESRC II, JBV 3, HHVBC, Raj 171, SDMV 90031, GB 8735, SDMV 95045, ICMS 7704, and RCB were found in the parentage of 48 of the R-lines in set-II (and none of those in set-I). Moreover, once the hybrid parents are developed, some of the promising ones amongst these first cycle parents are involved in the crossing programs to initiate another cycle of inbreeding and selection to derive new inbreds, as was reflected from set-II lines where 42 of the 88 B-lines assessed had 19 B-lines designated between 1992 to 2004 in their parentage. This approach of crossing elite inbreds is also practiced in maize to derive new inbreds [32] [33]. Contrary to our findings, reduction in the genetic diversity of new inbreds was found in comparison to the historical maize inbreds, in a study conducted on 8 new and 32 historical inbreds using 83 SSR markers [34].

\subsection{Within-Line Genetic Variability in the Hybrid Parents}

The levels of heterozygosity detected for most of the SSR primer pairs in B- and R-lines of both the sets was within acceptable limits $(<0.05)$, while it was high up to 0.12 in case of some primer pairs. A few SSR primer pairs detected heterozygosity in greater numbers of B- and R-lines than others. For instance, SSR locus Xpsmp2077 detected heterozygosity in 10 B-lines and 22 R-lines in set II. Likewise, some of the B- and R-lines were more heterozygous than others. For example, B-line (B-23) showed heterozygosity for eight SSR primer pairs while R-lines such as R-144 and R-160 were found heterozygous for 7 to 8 SSR primer pairs. The probable reason for high heterozygosity in some inbreds in comparison to others may be either due to 1) residual heterozygosity, 2) mutation and/or mutational bias at specific SSR loci, or 3) duplicate loci [35] [36]. Some level of within-line genetic variability has been reported earlier in advanced generation hybrid parents of pearl millet [37] [38]. Similar to these results, within-line variability has also been found in long-time bred maize inbreds for SSR markers [39] [40].

\subsection{Genetic Diversity between Seed and Restorer Parents and Its Linkage with Parentage}

ICRISAT follows a trait-based breeding approach to develop a phenotypically diverse range of hybrid parents to meet the diverse needs of various agro-ecologies, with high grain yield as a common desired trait. The traits used as selection criteria are based on regional preferences, which include various maturity types, plant height (grain vs. dual-purpose), tillering ability, panicle traits (length, thickness, and compactness), and seed traits (color and seed size). Seed parents (B-lines) are generally bred for short height $(<100 \mathrm{~cm})$ and for high grain yield component traits, while R-lines are generally bred for taller height $(150-180 \mathrm{~cm})$, more tillers, relatively smaller seed size, and profuse pollen production [41]. This unique differential trait requirement of B- and R-lines is met by involving quite diverse parents in each of these B- and R-line development programs. This distinctness in parentage and ideotypes was reflected in marker-based clustering pattern of set I and set II lines which clearly separated B-lines from R-lines in two clusters. This was also evidenced from combined AMOVA of both the sets, which indicated significant variation between B- and R-line groups. The grouping of B- and R-lines into two separate clusters was well detected by markers under study, as 27 of the 28 markers had significant Fst. This marker-based clustering pattern for these recently bred 166 set-II B- and R-lines, which separated most of the B- and R-lines into 2 separate clusters, was also reported earlier for ICRISAT-bred designated Band R-line hybrid parents [14], indicating existence of two diverse and broad-based pools in hybrid parents of pearl millet.

Genetic variation assessed for 166 newly developed hybrid parental inbreds (88 B-lines and 78 R-lines) using 28 SSR primer pairs detected an average gene diversity of 0.61 , indicating sufficient polymorphism to characterize this set of breeding lines for their genetic diversity. The clustering pattern of newly developed set-II Band R-lines further revealed that the B- and R-lines each grouped into four sub-clusters within their respective cluster, and sharing of a common parent can contribute to this clustering of inbreds in common sub-cluster. Se- 
venteen of the 22 B-lines in cluster B-I had 843B in their parentage, while 7 of the 15 lines in cluster B-II had involvement of both $843 \mathrm{~B}$ and $81 \mathrm{~B}$ in their parentage. Seed parental lines sharing $843 \mathrm{~B}$ in their parentage were scattered in all the four sub-clusters, with the maximum number being found in cluster B-I, which further supported earlier observation about clustering of most of the B-lines with 843B in their parentage in one large cluster [10] [14]. Also, 14 of 24 B-lines in cluster B-III had involvement of progeny from a single composite HHVBC, which was used as a source of thick-panicles in the crossing programs. Earlier, such clustering of thick-panicled B-lines derived from HHVBC, like ICMB 00555, ICMB 01222 and ICMB 01333 was observed in pearl millet [13]. Likewise, 4 of the 8 R-lines in cluster R-II had lines derived from ICRISAT's Smut Resistant Composite (SRC) in their parentage, while 13 of the 19 R-lines in cluster R-IV had either progeny from MRC (Mandore Restorer Composite), Rajasthan Composite Bajra (RCB) or Rajasthan Inbred Bajra (RIB), with each of these three parental composites having been constituted using breeding lines from the same arid parts of western India.

\subsection{Pattern of Genetic Diversity in Hybrid Parents and Breeding Implications}

Most of the 379 ICRISAT-Patancheru bred pearl millet B- and R-lines were found in their respective groups based on the clustering patterns detected by 28 SSR primer pairs, as 94\% of the B-lines and $84 \%$ of the R-lines were found in their respective B- and R-line clusters, indicating existence of two diverse and broad-based pools, one each representing seed and restorer parents. However, the variation between B-lines and R-lines has decreased over time as evident from decrease of variation between B- and R-lines from 16.98\% in set-I to 9.92\% in set-II. This narrowing down of variability between B- and R-lines might be due to involvement of certain common parents, both in seed parent (B-line) and restorer parent (R-line) breeding programs. Though, mostly a specific genetic stock is used either in the B-line or R-line crossing program based on its plant ideotype and fertility/sterility reaction, occasionally a particular line owing to its very specific trait is used simultaneously in development of both B- and R-lines. For instance, thick-panicle progenies derived from a B-composite (HHVBC) should be used only in the B-line program due to their closeness to B-plant ideotype, and also for having involvement of B-lines in its constitution. But owing to the shortage of donors for thick-panicle trait in R-line backgrounds, this line has been used occasionally in the R-line crossing program to introgress thick-panicle in R-lines. This approach has the risk of narrowing down the variability between B- and R-lines, as is reflected from the clustering of $23 \%$ of set-II R-lines (18 of 78 R-lines) with B-line clusters. Nine of these 18 R-lines had one or more B-lines in their parentage, of which 7 had involvement of a progeny from seed parent composite HHVBC. Also, of the 7 B-lines found clustered with R-lines, 2 had of progenies of a population ICTP 8202 in their parentage, a population with several restorer plant ideotype traits. Hence, efforts must be made to search for trait-specific donors separately in the B- and R-specific backgrounds for use in future crossing purposes, rather than continuing to use the same genetic stocks for a common trait in both of B- and R-line breeding programs. The separation of most of B- and R-lines into two separate clusters, in both previously designated and newly developed hybrid parents suggested that these two broad pools are continuously separated from each other, and B $\times$ B and R $\times$ R crosses should be made between and within set-I and set-II of B- and R-lines to generate diverse range of new recombinants for $\mathrm{B}$ - and R-line development.

The present study identified 15 B- and 37 R-lines from both the sets of lines with unique alleles (1 to 2 alleles) not present in other lines that can be used as genetic tags to supplement distinctness, uniformity, and stability (DUS) tests, thus enabling ICRISAT to protect these from possible infringement by multiple users who have received these lines for use in their hybrid breeding programs. Furthermore, presence of line-specific alleles may have association with some distinct trait(s) of the particular line, which merits further investigation. Moreover, the higher number of rare and unique alleles found among both B- and R-lines in this study suggests that these lines have been derived from a very diverse genetic base (which is in agreement with the diverse pedigrees of the lines included in this study).

\section{Conclusion}

The present study clearly differentiated newly developed hybrid parents bred by the pearl millet hybrid parents breeding program at ICRISAT-Patancheru from the previously designated hybrid parents bred at this location, indicating continuous involvement of new genetic stocks by the ICRISAT-Patancheru pearl millet hybrid parents breeding program, while maintaining consistent levels of genetic diversity among hybrid parents over time. 
The results indicated existence of two diverse and broad-based pools, one each for seed and restorer parents. Some decrease of genetic variability between B- and R-lines in the current set of inbreds was noted compared with those developed previously by this program. Hence, there is a need to identify trait-specific donors within the B- and R-line programs separately and to avoid using same donor in both of these programs. Significant diversity was observed within the pearl millet B- and R-line groups developed at ICRISAT-Patancheru, and they should be utilized to generate new wave of seed and restorer parents by involving lines from within the B-line and R-line groups and having greater genetic distances between them.

\section{Acknowledgements}

Authors acknowledge the support provided by ICRISAT-Sehgal Family Foundation Endowment Fund to conduct this study, as a part of project (YSFF06) "Molecular diversity in relation to morphological diversity and yield heterosis in sorghum and pearl millet”. Preparation of this publication has been undertaken as part of the CGIAR Research Program on Dryland Cereals.

\section{References}

[1] Andrews, D.J. and Anand Kumar, K. (1992) Pearl Millet for Food and Forage. Advances in Agronomy, 48, 89-139. http://dx.doi.org/10.1016/S0065-2113(08)60936-0

[2] Bonamigo, L.A. (1999) A cultura do milheto no Brasil, implantação e desenvolvimento no cerrado. In: Lopes de Farias Neto, A., Fernando Amabile, R., Martins Netto, D.A., Yamashita, T. and Gocho, H., Eds., Proceedings of the International Pearl Millet Workshop, Planaltina, 9-10 June 1999, Planaltina, D.F., Embrapa Cerrados, 31-65.

[3] Indiastat (2013) http://www.Indiastat.com/agriculture/2/agriculturalarealanduse/152/areaundercrops19502013/4484934/stats.aspx

[4] Yadav, O.P. and Rai, K.N. (2013) Genetic Improvement of Pearl Millet in India. Agricultural Research, 2, $275-292$. http://dx.doi.org/10.1007/s40003-013-0089-z

[5] ICRISAT (2012) Hybrid Parents Research Consortium. The Jewels of ICRISAT. International Crops Research Institute for the Semi-Arid Tropics (ICRISAT), Patancheru, 48-51.

[6] Senthilvel, S., Jayashree, B., Mahalakshmi, V., Kumar, P.S., Nakka, S., Nepolean, T. and Hash, C.T. (2008) Development and Mapping of Simple Sequence Repeat Markers for Pearl Millet from Data Mining of Expressed Sequence Tags. BMC Plant Biology, 8, 119. http://dx.doi.org/10.1186/1471-2229-8-119

[7] Yadav, O.P., Mitchell, S.E., Zamora, A., Fulton, T.M. and Kresovich, S. (2007) Development of New Simple Sequence Repeat Markers for Pearl Millet. SAT eJournal, 3.

[8] Rajaram, V., Nepolean, T., Senthilvel, S., Varshney, R.K., Vadez, V., Srivastava, R.K., Shah, T.M., Supriya, A., Kumar, S., Kumari, B.R., Bhanuprakash, A., Narasu, M.N., Riera-Lizarazu, O. and Hash, C.T. (2013) Pearl Millet [Pennisetum glaucum (L.) R. Br.] Consensus Linkage Map Constructed Using Four RIL Mapping Populations and Newly Developed EST-SSRs. BMC Genomics, 14, 159. http://www.biomedcentral.com/1471-2164/14/159 http://dx.doi.org/10.1186/1471-2164-14-159

[9] Moumouni, K.H., Kountche, B.A., Jean, M., Hash, C.T., Vigouroux, Y., Haussmann, B.I.G. and Belzile, F. (2015) Construction of a Genetic Map for Pearl Millet, Pennisetum glaucum (L.) R. Br., Using a Genotyping-by-Sequencing (GBS) Approach. Molecular Breeding, 35, 5. http://dx.doi.org/10.1007/s11032-015-0212-X

[10] Kapila, R.K., Yadav, R.S., Plaha, P., Rai, K.N., Yadav, O.P., Hash, C.T. and Howarth, C.J. (2008) Genetic Diversity among Pearl Millet Maintainers Using Microsatellite Markers. Plant Breeding, 127, 33-37.

[11] Sumathi, P., Sumanth, M., Vinodhana, N.K. and Sathya, M. (2013) Assessment of Genetic Distance among the Inbred Lines of Pearl Millet (Pennisetum glaucum (L.) R. Br.) Using SSR Markers. IJBAF, 1, 153-162.

[12] Rai, K.N., Gupta, S.K., Bhattacharjee, R., Kulkarni, V.N., Singh, A.K. and Rao, A.S. (2009) Morphological Characteristics of ICRISAT-Bred Pearl Millet Hybrid Seed Parents. Journal of SAT Agricultural Research, 7, 1.

[13] Gupta, S.K., Bhattacharjee, R., Rai, K.N. and Suresh Kumar, M. (2011) Characterization of ICRISAT-Bred Restorer Parents of Pearl Millet. Journal of SAT Agricultural Research, 9, 1-5.

[14] Nepolean, T., Gupta, S.K., Dwivedi, S.L., Bhattacharjee, R., Rai, K.N. and Hash, C.T. (2012) Genetic Diversity in Maintainer and Restorer Lines of Pearl Millet. Crop Science, 52, 2555-2563. http://dx.doi.org/10.2135/cropsci2011.11.0597

[15] Burton, G.W. (1968) Registration of Pearl Millet Inbreds Tift 23B 1 , Tift $23 \mathrm{~A}_{1}$, Tift 23DB 1 , and Tift 23D $\mathrm{A}_{1}$. Crop Science, 9, 397.

[16] Sharp, P.J., Kries, M., Sherry, P.R. and Gale, M.D. (1988) Location of $\beta$-Amylase Sequences in Wheat and Its Rela- 
tives. Theoretical and Applied Genetics, 75, 286-290. http://dx.doi.org/10.1007/BF00303966

[17] Qi, X., Pittaway, T.S., Lindup, S., Liu, H., Waterman, E., Padi, F.K., Hash, C.T., Zhu, J., Gale, M.D. and Devos, K.M. (2004) An Integrated Genetic Map and a New Set of Simple Sequence Repeat Markers for Pearl Millet, Pennisetum glaucum. Theoretical and Applied Genetics, 109, 1485-1493. http://dx.doi.org/10.1007/s00122-004-1765-y

[18] Applied Biosystems: GeneScan Analysis Software User Manual. Release 3.1 (1998) Applied Biosystems, Foster City.

[19] Applied Biosystems: Genotyper 3.1 (1998) Applied Biosystems, Foster City.

[20] Prasanth, V.P., Chandra, S., Jayashree, B. and Hoisington, D. (1998) AlleloBin-A Program for Allele Binning of Microsatellite Markers Based on the Algorithm of Idury and Cardon (1997). ICRISAT, Patancheru.

[21] Liu, K. and Muse, S.V. (2005) PowerMarker: Integrated Analysis Environment for Genetic Marker Data. Bioinformatics, 21, 2128-2129. http://dx.doi.org/10.1093/bioinformatics/bti282

[22] Li, Y.H., Guan, R.X., Liu, Z.X., Ma, Y.S., Wang, L.X., Li, L.H., Lin, F.Y., Luan, W.J., Chen, P.Y., Yan, Z., Guan, Y., Zhu, L., Ning, X.C., Smulders, M.J.M., Li, W., Piao, R.H., Cui, Y.H., Yu, Z.M., Guan, M., Chang, R.Z., Hou, A.F., Shi, A.N., Zhang, B., Zhu, S.L. and Qiu, L.J. (2008) Genetic Structure and Diversity of Cultivated Soybean (Glycine max (L.) Merr.) Landraces in China. Theoretical and Applied Genetics, 117, 857-871. http://dx.doi.org/10.1007/s00122-008-0825-0

[23] Upadhyaya, H.D., Dwivedi, S.L., Baum, M., Varshney, R.K., Udupa, S.M., Gowda, C.L.L., Hoisington, D. and Singh, S. (2008) Genetic Structure, Diversity, and Allelic Richness in Composite Collection and Reference Set in Chickpea (Cicer arietinum L.). BMC Plant Biology, 8, 106. http://dx.doi.org/10.1186/1471-2229-8-106

[24] Excoffier, L., Smouse, P. and Quattro, J. (1992) Analysis of Molecular Variance Inferred for Metric Distances among DNA Haplophytes: Applications to Human Mitochondrial DNA Restriction Data. Genetics, 131, 479-491.

[25] Perrier, X., Flori, A. and Bonnot, F. (2003) Data Analysis Methods. In: Hamon, P., Seguin, M., Perrier, X. and Glaszmann, J.C., Eds., Genetic Diversity of Cultivated Tropical Plants, Science Publishers, Enfield, 43-76.

[26] Rai, K.N., Andrews, D.J. and Rao, A.S. (1995) Registration of NCD2 Pearl Millet Germplasm. Crop Science, 35, 1237-1238. http://dx.doi.org/10.2135/cropsci1995.0011183X003500040102x

[27] Stegmeier, W.D., Andrews, D.J., Rai, K.N. and Hash, C.T. (1998) Pearl Millet Parental Lines 843A and 843B. International Sorghum and Millets Newsletter, 39, 129-130.

[28] Rai, K.N., Bidinger, F.R., Hussain, K. and Rao, A.S. (1998) Registration of ICMP 94001 Pearl Millet Germplasm. Crop Science, 38, 1411. http://dx.doi.org/10.2135/cropsci1998.0011183X003800050065x

[29] Anand Kumar, Andrews, D.J., Jain, R.P. and Singh, S.D. (1984) ICMA-1 and ICMB-1 Pearl Millet Parental Lines with A1 Cytoplasmic-Genic Male Sterility System. Crop Science, 24, 832. http://dx.doi.org/10.2135/cropsci1984.0011183X002400040066x

[30] Stich, B., Haussmann, B.I.G., Pasam, R., Bhosale, S., Hash, C.T., Melchinger, A.E. and Parzies, H. (2010) Patterns of Molecular and Phenotypic Diversity in Pearl Millet [Pennisetum glaucum (L.) R. Br.] from West and Central Africa and Their Relation to Geographical and Environmental Parameters. BMC Plant Biology, 10, 216. http://dx.doi.org/10.1186/1471-2229-10-216

[31] van Inghelandt, D., Melchinger, A.E., Lebreton, C. and Stich, B. (2010) Population Structure and Gentic Diversity in a Commercial Maize Breeding Program Assessed with SSR and SNP Markers. Theoretical and Applied Genetics, 120, 1289-1299. http://dx.doi.org/10.1007/s00122-009-1256-2

[32] Hallauer, A.R., Russell, W.A. and Lamkey, K.R. (1988) Corn Breeding. In: Sprague, G.F. and Dudley, J.W., Ed., Corn and Corn Improvement, 3rd Edition, Agronomy, American Society of Agronomy-Crop Science Society of AmericaSoil Science Society of America, Madison, 463-564.

[33] Troyer, A.F. (1990) A Retrospective View of Corn Genetic Resources. Journal of Heredity, 81, 17-24.

[34] Lu, H. and Bernardo, R. (2001) Molecular Marker Diversity among Current and Historical Maize Inbreds. Theoretical and Applied Genetics, 103, 613-617. http://dx.doi.org/10.1007/PL00002917

[35] Senior, M.L., Murthy, J.P., Goodman, M.M. and Stubber, C.W. (1998) Utility of SSRs for Determining Genetic Similarities and Relationships in Maize Using an Agarose Gel System. Crop Science, 38, 1088-1098. http://dx.doi.org/10.2135/cropsci1998.0011183X003800040034x

[36] Matsouka, Y., Mitchell, S.E., Kresovich, S., Goodman, M. and Dobeley, J. (2002) Microsatellite in Zea-Variability, Patterns of Mutations and Use for Evolutionary Studies. Theoretical and Applied Genetics, 104, 436-450. http://dx.doi.org/10.1007/s001220100694

[37] Hash, C.T., Thakur, R.P., Rao, V.P. and Bhaskar Raj, G. (2006) Evidence for Enhanced Resistance to Diverse Isolates of Pearl Millet Downy Mildew through Gene Pyramiding. International Sorghum and Millets Newsletter, 47, 134-138.

[38] Gupta, S.K., Nepolean, T., Rai, K.N., Hash, C.T., Bhattacharjee, R. and Rathore, A. (2012) Within-Line Genetic Variation for Quantitative Characters and SSRs in Long-Time Maintained Inbreds in Pearl Millet [Pennisetum glaucum (L.) 
R. Br.]. The European Journal of Plant Science and Biotechnology, 6, 109-113.

[39] Gethi, J.G., Labate, J.A., Lamkey, K.R., Smith, M.E. and Kresovich, S. (2002) SSR Variation in Important U.S. Maize Inbred Lines. Crop Science, 42, 951-957. http://dx.doi.org/10.2135/cropsci2002.0951

[40] Heckenberger, M., Van der Voort, J.R., Melchinger, A.E., Peleman, J. and Bohn, M. (2003) Variation of DNA Fingerprints among Accessions within Maize Inbred Lines and Implications for Identification of Essentially Derived Varieties: II. Genetic and Technical Sources of Variation in AFLP Data and Comparison with SSR Data. Molecular Breeding, 12, 97-106. http://dx.doi.org/10.1023/A:1026040007166

[41] Rai, K.N., Kulkarni, V.N., Thakur, R.P., Haussmann, B.I.G. and Mgonja, M.A. (2006) Pearl Millet Hybrid Parent’s Research: Approaches and Achievements. In: Gowda, C.L.L., Rai, K.N., Belum, Reddy, V.S. and Saxena K.B., Eds., Hybrid Parents Research at ICRISAT, International Crops Research Institute for the Semi-Arid Tropics, Patancheru 502 324, Andhra Pradesh, 11-74. 